Mots. Les langages du politique

$73 \mid 2003$

Les discours de la guerre

\title{
Les méditations militaires de Scipione Ammirato Guerre et raison d'État
}

\section{Michel Senellart}

\section{(2) OpenEdition}

\section{Journals}

Édition électronique

URL : https://journals.openedition.org/mots/16932

DOI : $10.4000 /$ mots. 16932

ISSN : 1960-6001

Éditeur

ENS Éditions

\section{Édition imprimée}

Date de publication : 1 novembre 2003

Pagination : 161-168

ISBN : 2-84788-043-7

ISSN : 0243-6450

Référence électronique

Michel Senellart, « Les méditations militaires de Scipione Ammirato Guerre et raison d'État », Mots. Les langages du politique [En ligne], 73 | 2003, mis en ligne le 13 octobre 2008, consulté le 23 avril 2022.

URL : http://journals.openedition.org/mots/16932 ; DOI : https://doi.org/10.4000/mots. 16932 


\section{Les méditations militaires de Scipione Ammirato Guerre et raison d'État}

La raison d'État, dans son acception historique, désigne cet ensemble de doctrines, aux $17^{\mathrm{e}}-18^{\mathrm{e}}$ siècles, qui posent le problème de la conservation de l'État non dans l'hypothèse d'une genèse autarcique ni sur l'horizon d'une universalité pacifiée, mais dans un contexte de concurrence, de lutte et d'insécurité générale. À ce titre, il est incontestable qu'elle a affaire à la guerre, sinon comme à son principal objet, du moins comme à son souci le plus grave. Il semble donc légitime de se demander quelle place elle lui accorde et quelles limites elle lui fixe. Poser la question en ces termes, cependant, ne laisse pas de faire problème. Une telle question, en effet, - comment la raison d'État justifie-t-elle la guerre ? - présuppose que la raison d'État constitue le cadre théorique à l'intérieur duquel se serait développée une nouvelle analyse de la guerre, différente de celle, élaborée depuis le Moyen Âge, par la littérature théologico-juridique. Or s'il est exact que, dans cette tradition, la construction d'un cadre théorique général, tirant sa spécificité de principes universels, précédait la mise en question de la guerre comme phénomène particulier ${ }^{2}$, il n'est pas sûr qu'il en aille de même dans le discours de la raison d'État. Loin que ce discours définisse le cadre à l'intérieur duquel s'énoncerait une nouvelle pensée de la guerre, on peut se demander si ce n'est pas celle-ci, à l'inverse, qui a défini l'espace à partir duquel ce discours est devenu possible. Ce serait moins la raison d’État, en somme, qui justifierait la guerre, selon tel ou tel critère de réalisme, que la guerre qui, dans sa logique propre, expliquerait la raison d'État. La distinction des deux points de vue n'est pas purement académique. Elle oppose deux types de rationalité, l'une normative, en termes de droit (quid juris ?), l'autre instrumentale, en termes de moyens (comment faire ?). La première correspond sans doute à tout un ensemble de textes, à l'instar du Discorso della ragione di guerra de G. Frachetta ${ }^{3}$ qui distingue deux formes du jus belli, la guerre

1. ENS-LSH - 15, Parvis René-Descartes, 69366 Lyon cedex 07-msenell@club-internet.fr

2. Voir par exemple Thomas d'Aquin, Somme théologique, IIa-IIae, qu. 40.

3. Dans 1592, L'Idea del libro de'governi di stato et di guerra, Venise, f. $47 \mathrm{v}-63 \mathrm{v}$. 
juste et la guerre licite 4 , et deux formes de ratio belli, la vraie et l'apparente. Malgré les audaces dont ils font preuve par rapport au modèle scolastique, ces textes ne représentent peut-être pas la tendance la plus novatrice du discours de la raison d'État. Ils ne doivent pas dissimuler en tout cas l'émergence d'une autre tendance, plus radicale, consistant non à subsumer la pratique de la guerre sous les maximes de la prudence politique, mais à redéfinir celle-ci à partir de la manière, historiquement liée à l'évolution des techniques et des stratégies, dont on fait désormais la guerre. Renversement capital dans la notion de lois de la guerre. Alors qu'au Moyen Âge, les lois de la guerre étaient les lois imposées à la guerre, pour la contenir dans les limites juridico-morales de la guerre juste, à partir du $16^{\mathrm{e}}$ siècle, elles deviennent les lois imposées par la guerre, en dehors de toute normativité juridique et religieuse. C'est chez Guicciardini, sans doute, que s'affirme le plus nettement cette conscience du bouleversement des règles du jeu politique par l'irruption de la guerre moderne, fondée sur l'offensive massive et foudroyante d'une armée disposant d'une puissante artillerie ${ }^{5}$.

Mon intention, ici, n'est pas d'esquisser une interprétation générale de la raison d'État à partir des profonds bouleversements de la technique militaire au $16^{\mathrm{e}}$ siècle $^{6}$. La question que je voudrais poser est plus étroitement circonscrite. Elle procède d'abord d'un problème de méthode. De Meinecke et de Mattei aux travaux les plus récents ${ }^{7}$, on étudie les variations de l'idée de

4. «Guerre licite est celle, quand quelqu'un n'ayant point de juste, ni de légitime sujet de guerroyer, pense néanmoins y être bien fondé après avoir fait faire un diligent état, et soigneux examen de ses raisons » (trad. de L. Melliet, dans : [Ammirato], 1633, Discours politique et militaire sur C. Tacite, Rouen, p. 895-896. Sur cet ouvrage, dont la $1^{\text {re }}$ édition parut en 1628, voir M. Senellart, 1997, « La traduction des Discorsi d'Ammirato par L. Melliet (1628) : déplacements, additions, reconstruction », dans G. Dottoli (dir.), Politique et littérature en France aux XVIe et XVII siècles, Bari, Adriatica-Paris, Didier, p. 273-290).

5. Voir F. Guicciardini, 1561, Histoire d'Italie, trad. fr. de J.-L. Fournel et J.-C. Zancarini, 1996, Paris, Robert Laffont, « Bouquins ». " Ainsi était entrée en Italie, écrit-il à propos de l'invasion par les troupes françaises de Charles VIII en 1494, une flamme, une peste, qui non seulement changea les États, mais aussi la façon de les gouverner et les façons de faire la guerre ».

6. Sur ces bouleversements, voir notamment la bonne synthèse d'A. Campillo, 1986, La fuerza de la razon. Guerra, esatdo y ciencia en los tratados militares del Rinacimiento, de Maquiavelo a Galileo, Secretariado de publicaciones, Universidad de Murcia.

7. F. Meinecke, 1924, Die Idee der Staatsräson in der neueren Geschichte, trad. de M. Chevallier, 1973, L'idée de la raison d'État dans l'histoire des Temps modernes, Genève, Droz ; R. de Mattei, 1979, Il problema della « ragion di Stato " nell'età della controriforma, Milan-Naples, Riccardo Ricciardi. Pour la bibliographie des travaux récents, voir M. Senellart, 1997, «Le problème de la raison d'État de Botero à Zuccolo (15891621) », dans Ch. Menasseyre et A. Tosel (dir.), Figures italiennes de la rationalité, Paris, Kimé, p. 153-189. 
raison d'État sur la base d'énoncés réunis par leur commune appartenance à un même discours, sans se demander, trop souvent, dans quel genre spécifique ils s'inscrivent, quelle place ils y occupent, quel rôle ils y jouent, à quels lecteurs ils s'adressent ni à quelle efficacité ils prétendent. C'est ainsi que l'on analyse la définition de la raison d'État formulée par Scipione Ammirato $^{8}$ en isolant le bref traité qu'il lui consacre de l'ensemble du dispositif argumentatif dans lequel il s'insère et dont il forme une pièce certes centrale, mais non autonome - son commentaire des Annales et des Histoires de Tacite $^{9}$. Or, je me demande si l'on ne se condamne pas, de la sorte, à méconnaitre sa véritable signification. La définition célèbre de $\mathrm{S}$. Ammirato - la raison d'État comme contravention, ou dérogation, aux lois ordinaires en vue de l'intérêt commun ${ }^{10}$ - est fréquemment interprétée en termes de recodification juridique des pratiques de puissance, au sein d'une hiérarchie de "raisons » ou lois - loi naturelle, loi civile, droit de guerre, droit des gens, loi divine qui structurent le réel. Réduite au principe juridique de la dérogation ${ }^{11}$, la «bonne raison d'État» permettrait ainsi de concilier, non sans ambigüité, exigences politiques, normes juridiques et impératifs éthiques. Une telle présentation, cependant, a l'inconvénient majeur de faire silence sur ce qui constitue, pour S. Ammirato, la principale source de la prudence politique: l'histoire, dans sa pure factualité. C'est contre les juristes, et non sur leur terrain, que S. Ammirato défend l'idée de deroga, afin d'ouvrir le champ d'une science historique du gouvernement :

Que personne ne cuide mieux apprendre les affaires d'État [le cose di stato] d'aucun livre que de l'histoire, parce que les excellents historiographes sont ceux qui, non en la spéculation, ains au fait [non in speculazioni, ma in fatto] et, comme

8. Publiciste et historien florentin, Scipione Ammirato (1531-1600) est le principal représentant du tacitisme italien à la fin du $16^{\mathrm{e}}$ siècle, courant de pensée qui constitue une sorte de double, plus ou moins masqué, du machiavélisme, sans se réduire toutefois aux maximes de ce dernier. Il est, avec Giovanni Botero, l'un des premiers théoriciens de la ragion di stato. Pour plus de précisions sur cet auteur, voir R. de Mattei, 1963, Il pensiero politico di Scipione Ammirato, Milan, Giuffrè, et M. Senellart, 1997, « La critique de Machiavel dans les Discorsi sopra Tacito (1594) d'Ammirato », Problèmes d'histoire des religions, t. VIII, Bruxelles, Éditions de l'Université de Bruxelles, p. 105-119.

9. 1594, Discorsi sopra Cornelio Tacito, Florence. Nombreuses rééditions jusqu'au $19^{\mathrm{e}}$ siècle. Sur les traductions françaises de cet ouvrage au $17^{\mathrm{e}}$ siècle, voir note 3 . Celle de Melliet, que je cite désormais, modifie l'ordre des chapitres.

10. Ibid., XII, 1 ; trad. de L. Melliet, VI, 7, p. 338 : « [La] raison d'État n'[est] autre chose qu'une contravention aux raisons ordinaires pour le respect du bien public, ou pour le respect d'une plus grande et plus universelle raison $»$.

11. Sur cette interprétation de la raison d'État en termes de dérogation, voir M. Viroli, 1992, From Politics to Reason of State. The Acquisition and Transformation of the Language of Politics 1250-1600, Cambridge UP, « Ideas in context ». 
on dit, en argent comptant et pécune nombrée [in pecunia numerata], donnent les préceptes, les règles et les enseignements des accidents, des évènements, des fortunes mêlées qui se rencontrent tous les jours au gouvernement des États, soit monarchiques, aristocratiques ou populaires, et qui traitent des affaires de paix et de guerre, d'alliances, de confédérations, et de toutes les affaires politiques et militaires, autrement [dit] publiques. Et quand je multiplie les exemples, je ne le fais à autre fin, que pour montrer la vérité et uniformité de cette doctrine ${ }^{12}$.

Avec S. Ammirato, à la suite de Machiavel, s'affirme un genre nouveau, distinct aussi bien des Institutions du prince, des recueils d'exempla historiques et des florilèges de maximes que des traités de droit public et de science politique : le commentaire historico-politique. L'essor de ce genre se confond en partie avec le développement du tacitisme, mais ne s'y réduit pas, de même que ce dernier a des contours plus larges, ou plus flous, que le genre en question. Son étude reste encore pour une grande part à effectuer. Selon la classification établie par Arnaldo Momigliano ${ }^{13}$, on peut distinguer trois groupes d'écrits tacitistes, correspondant à leur chronologie même, du $16^{\mathrm{e}}$ au $17^{\mathrm{e}}$ siècle : celui des recueils d'aphorismes, à la manière de Carlo Pasquali, ou Charles Paschal ${ }^{14}$, traitant le texte tacitien comme une mine de sententiae, mais sans souci d'application pratique ${ }^{15}$; celui des commentaires politiques proprement dits, le groupe le plus prolifique, imprégné d'un machiavélisme plus ou moins diffus, qui commence avec S. Ammirato et se prolonge jusqu'au $18^{\mathrm{e}}$ siècle $^{16}$; celui, enfin, plus tardif et dérivé, des « traités systématiques consacrés aux lieux communs traditionnels, composés exclusivement ou presque de maximes tacitiennes $»$. Le commentaire politique se distingue donc par son effort pour articuler le récit historique à l'analyse de l'histoire présente, afin

12. Ouvr. cit., III, 13, p. 130-131, cité dans la trad. de Melliet, IV, 2, p. 203-204. Sur ce même thème, voir XIII, 4, p. 270-271 (trad. Melliet, VII, 3, p. 407-408), où S. Ammirato oppose le filosofo politico, versé dans la pratique de l'histoire, aux dottori di legge. Sur ce conflit, voir R. de Mattei, 1982, "La vertenza tra "filosofi politici" e "legisti" ", dans Il pensiero politico italiano nell'età della controriforma, Milan-Naples, R. Ricciardi, t. I, p. 164-187.

13. A. Momigliano, 1983, «Le premier commentaire politique de Tacite», dans Problèmes d'historiographie ancienne et moderne, Paris, Gallimard, "Bibliothèque des Histoires », p. 223-225.

14. 1581, C.C. Taciti ab excessu divi Augusti Annalium libri quattuor priores, et in hos observationes, Paris, apud R. Colombellum. Voir K. C. Schellhase, 1976, Tacitus in Renaissance Political Thought, Chicago UP, p. 122-123.

15. K. C. Schellhase, ouvr. cit., p. 123 : « He made no effort comparable to Machiavelli's to combine what he read in Tacitus with what he had learned from his own experience ».

16. Voir Th. Gordon, 1728, The Annales, to which are prefixed Political Discourses, Dublin. 
d'en dégager des maximes de conduite et d'action. Commentaire, à cet égard, est un mot trompeur, car c'est le présent historique qui forme sa véritable matière, éclairée ou rendue intelligible par l'enseignement tacitien. À l'inverse du commentaire critique, le commentaire historique ne vise pas à rétablir le passé dans sa forme authentique, mais à faire ressortir, dans la trame des évènements actuels, l'image d'une expérience déjà vécue et réfléchie.

L'hypothèse que je voudrais formuler est la suivante : le commentaire historico-politique ne constitue-t-il pas la forme d'un discours qui ne part pas de prémisses universelles, comme le discours théologique ou juridique, mais des déterminations actuelles de l'action politique, en les référant non à des critères invariables, mais à des situations comparables ? Plus précisément : n'est-ce pas parce qu'il pense la politique à partir des rapports de guerre qui déterminent le présent qu'il est voué à une radicale historicité, la guerre obligeant à regarder les choses non in speculazioni, ma in fatto et à payer ses conseils « en argent comptant»? Pour tenter de répondre à ces questions, il faut rappeler, tout d'abord, quelle guerre hante les Discours de S. Ammirato. Avec un grand nombre de ses contemporains, à commencer par Botero, il est obsédé par le péril turc ${ }^{17}$. «L'observation des choses politiques, écrit-il, nous enseigne qu'en l'espace de cent ans le Turc se peut faire maitre de l'Italie, s'il n'y est pourvu ${ }^{18} \gg$. De même qu'un bon artisan prévoit ce qui est nécessaire à son art et y pourvoit de bonne heure, de même l'art politique doit-il s'employer à prévenir cette menace qui, à travers l'Italie, pèse sur toute la chrétienté. La contradiction principale de son temps n'étant pas au sein du monde chrétien, mais entre celui-ci et l'islam, il importe de réunir toutes les forces en vue d'une nouvelle croisade sur terre et sur mer. Ce thème relativement banal s'articule, chez S. Ammirato, à un argument politique original. Il soutient en effet, contre Machiavel, que la division de l'Italie n'est pas due aux papes et que l'unité italienne, si elle avait été réalisée, aurait apporté plus d'inconvénients que d'avantages à l'Italie ${ }^{19}$. La multitude des principautés, avec des centres fortifiés répartis tout au long de la péninsule, lui parait une digue plus efficace qu'un prince unique contre l'envahisseur turc. Politique et religion se soutiennent ainsi l'une l'autre, la religion comme facteur de

17. Sur l'analyse par S. Ammirato du problème turc et les remèdes qu'il préconise, voir A. Alberti, 1931, "Politica e ragion di Stato nell' opera di Sc. "Ammirato" ", Atti dell' Accademia delle Scienze di Torino, Turin, classe di scienze morali, stor. e filol., vol. LXVI, p. $620-625$.

18. Ouvr. cit., XII, 3 ; trad. L. Melliet, VI, 9, p. 362.

19. Thèses défendues dans certains des Opuscoli publiés à Florence en 1637, longtemps après sa mort. Voir R. de Mattei, 1961, « Ammirato », Dizionario biografico degli Italiani, Rome, Istituto della Enciclopedia italiana, p. 3. 
solidarité politique entre les États, la politique comme facteur de renforcement de l'unité religieuse. Relation circulaire, la politique trouvant sa limitation dans ce qui lui permet, en fait, d'accroitre son efficace contre l'ennemi commun. C'est sur fond de cette guerre latente, tendue vers un affrontement décisif, que se comprend le concept de ragion di stato employé par S. Ammirato. L'ambivalence qui le caractérise - raison transgressant toutes les raisons ordinaires, mais subordonnée à la loi divine - ne tient pas à l'inconsistance de l'esprit de compromis, mais à la structure même du rapport de forces qui met en jeu, face à l'essor ottoman, l'avenir du monde chrétien.

À propos de la famine, ce péril intérieur qui menace chaque État, S. Ammirato écrit qu'il est nécessaire, pour y remédier, « d'imiter l'ordre de la guerre », en imposant aux villes ${ }^{20}$ le modèle du camp :

Non content que la cité soit partie et subdivisée en plusieurs parties, [il faudrait] créer des chefs de rue, lesquels comme sergents de bande, qui obéissent à leur capitaine, auront le soin des habitants de leur rue, donneront de la besogne aux pauvres $^{21}$ et par conséquent procureront pour eux qu'ils aient à manger ${ }^{22}$.

La lutte contre la famine impose la suspension des relations civiles ordinaires, l'établissement d'une discipline rigoureuse, l'usage de la contrainte pour le salut commun. Il en va de même pour le péril extérieur que constitue l'expansion ottomane, à ceci près toutefois que si la famine est un accident, la menace turque, quant à elle, est permanente. La raison d'État autorise à prendre des dispositions extraordinaires dans les cas extrêmes. Elle est donc, en quelque sorte, la pointe de l'art politique qui consiste à prévoir ces cas et à s'y préparer. Ou plutôt, elle est le prix à payer lorsque surviennent de tels cas, sans qu'on ait eu le temps d'y pourvoir à l'avance. Ainsi, en cas de famine, si la discipline ne suffit pas et que l'urgence l'exige, la mortalité des sujets n'arrivant jamais sans grand dommage du prince ${ }^{23}$, lui est-il permis de réquisitionner le blé des riches et même, en dernier recours, de vendre l'argenterie des églises ${ }^{24}$. Le péril turc est un cas extrême dont les princes

20. Mais cette disposition s'applique également aux campagnes : « Il faut que le bon ordre tenu en la cité soit aussi observé aux champs » (ouvr. cit., XII, 3 ; trad. L. Melliet, VI, 9, p. 373).

21. C'est-à-dire : « ordonneront l'exercice, le travail et le métier en quoi les souffreteux devront être occupés, soit pour rendre moins onéreuse et griève la libéralité de ceux qui les soulageront, soit pour dompter la truandise et combattre l'oisiveté » (ibid., trad. Melliet, p. 372).

22. Ibid., p. 372.

23. Ibid., p. 373.

24. Voir XII, 1 ; trad. Melliet, VI, 7, p. 353 ; voir également XV, 5 (trad. Melliet, VIII, $10)$, où sont précisées les limites de ce droit. 
d'Europe négligent de se soucier, leurs querelles étant cause que «nous laissons croitre la puissance du Turc, et nous consommant nous-mêmes, nous rendons faibles et impuissants pour résister à ses nombreuses et épouvantables armées, si jamais il lui prend volonté de venir nous attaquer ${ }^{25}$ ». Or « un prince n'est pas sage qui n'a de bonne heure assemblé des nerfs pour la guerre, afin d'être bien préparé aux extrêmes cas que le temps peut faire naitre $^{26} »$. Problème économique, auquel S. Ammirato consacre plusieurs discours $^{27}$, mais également militaire : comment créer une armée disciplinée, fondée sur le modèle de la "sévère milice " des anciens, capable de faire pièce à la puissance du Turc ? Celui-ci, en effet, n'est pas simplement un ennemi cruel, perfide et inhumain ${ }^{28}$. Il est aussi un exemple de discipline martiale. Sa force réside moins dans ses troupes innombrables que dans ses régiments de janissaires soumis, dès leur jeune âge, à l'entrainement le plus rigoureux ${ }^{29}$. L'éthos disciplinaire qui traverse les Discorsi de S. Ammirato, et qui anime sa conception de la ragion di stato, le prince devant ordonner sa volonté propre à la considération du bien public, ne procède donc pas de la seule nostalgie de l'ordre romain. Il répond à un impératif stratégique, qui découle de la nature même de l'ennemi auquel sont confrontés les États italiens.

Loin donc que la raison d'État définisse les conditions, les règles et les fins de la guerre, il me semble que c'est la guerre qui dessine le champ de la raison d'État. Non pas la guerre allégorique des appétits et des passions ni celle, intemporelle, des rivalités entre voisins, mais la guerre comme structure historiquement déterminée du rapport des forces, dont dépend le maintien des équilibres établis. De ce point de vue, on peut dire que la raison d'État relève moins d'un discours éthico-religieux, caractérisé en termes de droit (quid juris ?), que d'un discours historico-politique, caractérisé en termes de fait. Que faire dans la situation qu'imposent les faits ? C'est cette question qui fait l'objet de ce que S. Ammirato appelle les «méditations militaires ${ }^{30} »$ :

Ainsi devrions-nous aller incessamment méditant, avec quelles armes il serait meilleur s'opposer à la puissance du Turc, avec quel ordre de bataille, avec quel mélange d'armes et de nations, avec quel nombre de gens à pied et à cheval, en quel temps, en quel lieu, avec quel avantage, quelle ruse et nouveau stratagème.

25. Ibid., I, 6 ; trad. Melliet, I, 6, p. 40.

26. Ibid., I, 11 ; trad. Melliet, I, 11, p. 60.

27. Voir ibid., I, 11, «Des finances militaires »; III, 8 (trad. Melliet, III, 9), « Des moyens d'augmenter les finances »; XVIII, 9 (trad. Melliet, X, 8), "Que les deniers sont les nerfs de la guerre $»$.

28. Voir ibid., XIII, 11 ; trad. Melliet, VII, 10, p. 457-458.

29. Ibid., XX, 2 ; trad. Melliet, XI, 9, p. 789.

30. Ibid., XIV, 2 ; trad. Melliet, VII, 12. 
Au défaut de quoi, nous connaitrons trop tard notre négligence, paresse et lâcheté touchant les affaires de la guerre ${ }^{31}$.

Méditation : S. Ammirato emploie le mot dans son double sens, qui correspond au grec meletê, de réflexion et d'exercice. «L'exercice est une action qui met en œuvre ce qu'on a appris en travaillant le corps, et la méditation fait le même effet, remémorant souvent avec l'entendement la chose qui a été enseignée. Tellement qu'on pourrait dire que la méditation est un exercice de l'esprit, comme l'exercice est une méditation du corps ${ }^{32} \gg$. La méditation surmonte ainsi l'opposition de la théorie et de la pratique. Elle est à la fois l'une et l'autre, remémoration, exercice du jugement, mise à l'épreuve. Les Discorsi de S. Ammirato ne doivent-ils pas se lire comme l'illustration d'un nouveau genre, issu de l'expérience des guerres modernes : celui des méditations politico-militaires ? Genre étranger à la pure spéculation, car si les méditations militaires, selon le mot que $\mathrm{S}$. Ammirato prête à Flavius Josèphe, sont des batailles sans sang (battaglie senza sangue), les batailles en revanche sont des méditations sanglantes (meditazioni con sangue) ${ }^{33}$.

31. Ibid. ; trad. Melliet, p. 469.

32. Ibid. ; trad. Melliet, p. 463.

33. Ibid. Voir Flavius Josèphe, 1980, Guerre des juifs, III, 75, trad. d'A. Pelletier, Paris, Les Belles Lettres. Josèphe utilise le mot meleté qui signifie « exercices, entrainements, manœuvres » et aussi, dans les dictionnaires anciens comme celui d'Estienne, méditations («On ne se tromperait pas en disant que leurs manœuvres (meletas) sont des batailles sans effusion de sang et que leurs batailles ne sont que des manœuvres avec effusion de sang »). Je remercie Christophe Batsch pour la référence précise de ce passage. 